\title{
Awareness of Cancer Pain Management among Nurses in an Albanian Oncologic Hospital
}

\author{
Zamira Imeraj \\ Faculty of Technical Medical Sciences, University of Medicine, Tirana, Albania \\ Arianit Kokobobo \\ Oncologic department, University Hospital Centre "Mother Theresa", Tirana, Albania \\ Enkelejda Shkurti \\ Faculty of Technical Medical Sciences, University of Medicine, Tirana, Albania
}

\section{Doi:10.5901/jesr.2015.v5n2p35}

\section{Abstract}

The doctor-patient percentage in Albania as said by World Health Organization (WHO). In 2013, this ratio was 1,2:1000 in contrast the average 1:600, although that of nurse-patient is enhanced, 1:40 compared to WHO standard of 1:6. Occurrence of cancer is universally increasing and the mainstream of the complex cases arise in the developing countries like Albania. Nurses engage in a significant position in efficient ache administration since they have further connection with patients in any healthcare location, appraise the pain and its collision on the patients and relatives, therefore commence acts to supervise the pain. This survey is intended at evaluating the phase of awareness of cancer pain administration between skilled nurses at Oncologic department of University Hospital Centre "Mother Theresa" in Albania. Materials and Methods: Qualified nurses who approved to participate in the survey were examined applying self-administered questionnaire. The surveys comprised 3 main parts that is (a) Demography, (b) Pain appraisal, (c) Cancer Ache administration. The degree of comprehension of cancer pain administration was subsequently evaluated from their reaction to the questionnaires. 63 nurses fulfilled the questionnaire. 54 (85.71\%) were twofold trained. About 11 (17.4\%) participants had supplementary official instruction in ache management. 50 (79.36) respondents provided right meaning of pain ;only 3 (3\%) could confer a good explanation on the administration of cancer pain. 17 (26.9 \%) of the participants could apply Visual Analogue Scale (VAS) for ache estimation. Merely 12 (19\%) could affirm the application of opioids in cancer pain administration. This survey exposed insufficient comprehension in cancer ache management between the nurses. To attain holistic care for cancer individuals in all health care settings, further preparation in pain management is needed.

Keywords: awareness, cancer ache management, skilled nurses

\section{Introduction}

Pain is an awful sensory and expressive occurrence that is habitually linked with probable tissue injure (Merskey \& N. Bogduk 1994). Pain is a main health grumble by patients in the majority of health care settings. Ache administration requires a diverse team approach but in a developing country like Albania, not all nurses are well-informed about ache administration. Nurses are main actors in pain administration.

With the purpose of sustaining the standard of patients' care in health care centers, nurses should acquire adequate information to improve their contribution in the management of cancer ache in our patients. The proportion of nurses to patients of 1:40 is even superior than that of doctors to patient, conversely WHO average is 1nurse to 6 patients. Cancer pain has turned into a key health alarm, since of the increasing occurrence of cancer, regrettably approaching in delayed phases in developing countries like Albania.

For appropriate administration of cancer ache in patients, a care supplier is supposed to have sufficient knowledge of the kind of pain disorder and excellent pain evaluation abilities. There are two groups of pain specifically nociceptive and neuropathic pains.

Nociceptive pain is related to the reply of the nervous system to tissue injure. Conditional on the setting and indicators of the hurting incentive, nociceptive pain is extra separated into two: somatic and internal organs pain. 
Somatic pain is typically represented as a well-concentrated, sharp (knife-resembling) and hurting; whereas viscera ache is hard to define. Physical pain is correlated with injure to the skin, muscle and connective tissues, while viscera pain is related with spasming and boring ache (Merskey \& N. Bogduk 1994). Non-nociceptive pain covers mutually the neuropathic and psychological pain conditions. In both cases, nervous system reactions are uncommon. Neuropathic pain could arise without evident tissue injure.

The sort of pain suffered by tumor patients is compound multifaced in nature. Pain is the main frightened sign allied with tumor diseases and it is frequently found in nearly $70 \%$ of cancer individuals (Besson JM \& Chaouch A 2005). Tumor ache is generally related with cancer expansion and the force it exercises on the neighboring tissues and nerves. The snowballing outcome of processes undergone by tumor patients in the itinerary of cancer administration may cause pain.

There are diverse patterns of ache management and the result of every application relies on the expertise used by the care giver, particularly the nurses who occupy a chief task in patient's care in the majority of health care clinics, specifically at the primary health care centers in rural regions.

Somatic ache would be efficiently handled with opioids drugs while, neuropathic pain is best handled with anticonvulsant or antidepressant medicines. To participate significantly in pain administration, skilled nurses are supposed to own sufficient information to manage dissimilar kinds of pain in patients. The job of nurses in patient care is dissimilar and many-sided. Ache management is an essential element of patient's care and nurses are the bed rock.

Consequently, the task of nurses in pain administration cannot be over highlighted. They are dynamic contributors in the whole medical procedures of ache management. The course of tumor pain administration comprise the appraisal with Visual Analogue Scale (VAS) of 0 - 10 (where 0 - 3 means gentle ache; $4-7$ means reasonable and $8-10$ means harsh pain) or Verbal Rating Scale (VRS) scored as mild, moderate and severe. This is needed for the preparation of administering mechanism/medication and actual managing of the selected drug. The nurses also contribute in the assessment of pain treatment, thus the necessity for nurses to have high-quality knowledge of ache administration.

\section{Objectives}

This survey is to appraise the degree of proficiency in tumor ache management between the skilled nurses in a university hospital, where the top hands between nurses are typically preserved to care for still rising the occurrence of complex tumor cases, circumstances in which $74 \%$ of patients present with one type of ache or the other.

\section{Methodology}

The aim of the survey was clarified to the nurses and each of them was provided a set of questionnaires to fulfill. The questionnaire comprised 3 major parts, (a) Demography, under this title information about supplementary education I prerequisites were asked, (b) Pain estimation techniques, with stress on visual analogue level, verbal evaluation level (c) Processes of cancer pain administration, issues on pharmacological and physical therapy alternatives were inquired and questions were as well asked on role of opioids particularly oral morphine in cancer ache.

The questionnaires having nearly15 stem questions have been formerly pilot assessed. They were made to comprehend that contribution was chosen. Subsequent to a period of one month, out of 63 nurses on altered functions in nearly seven diverse areas, who assembled the criteria for options, one hundred and nineteen (63) returned their fulfilled questionnaires and these were investigated.

\section{Results}

The contributors who presented completed questionnaires were 63 nurses. These involved 58 (92\%) females and $5(8 \%)$ males. They were between 35-70 years old. Mainly 54 (85\%) of the participants had dual prerequisites: scheduled nurse (SN) and registered midwife (RM). Their period of medical nursing skill varied from 12-25 years. The data are illustrated in Table 1. There were 31 unfinished questionnaires, each one from female nurses with analogous age variety 35-70 year. 
Table 1. The demographic data and training Information of Respondents

\begin{tabular}{|c|c|}
\hline \multicolumn{2}{|c|}{ Number of Respondent (\%) } \\
\hline \multicolumn{2}{|l|}{ Gender status: } \\
\hline Male & $5(8 \%)$ \\
\hline Female & $58(92 \%)$ \\
\hline \multicolumn{2}{|l|}{ Age (years): } \\
\hline Range & $35-70$ \\
\hline Modal age range & $35-46$ \\
\hline Period of clinical Nursing experience (years) & $12-25$ \\
\hline \multicolumn{2}{|l|}{ Additional training (formal) in pain management } \\
\hline \multicolumn{2}{|l|}{ Acquired Training: } \\
\hline General Nursing and midwifery & $54(85.07 \%)$ \\
\hline (Additional training) Pain management & $8(12.6 \%)$ \\
\hline
\end{tabular}

Table 2 demonstrated that besides the common nursing certificate obtained by all the participants, $8(12.6 \%)$ of them had supplementary preparation in tumor pain administration.

Table 2. Reply to tumor pain appraisal/management

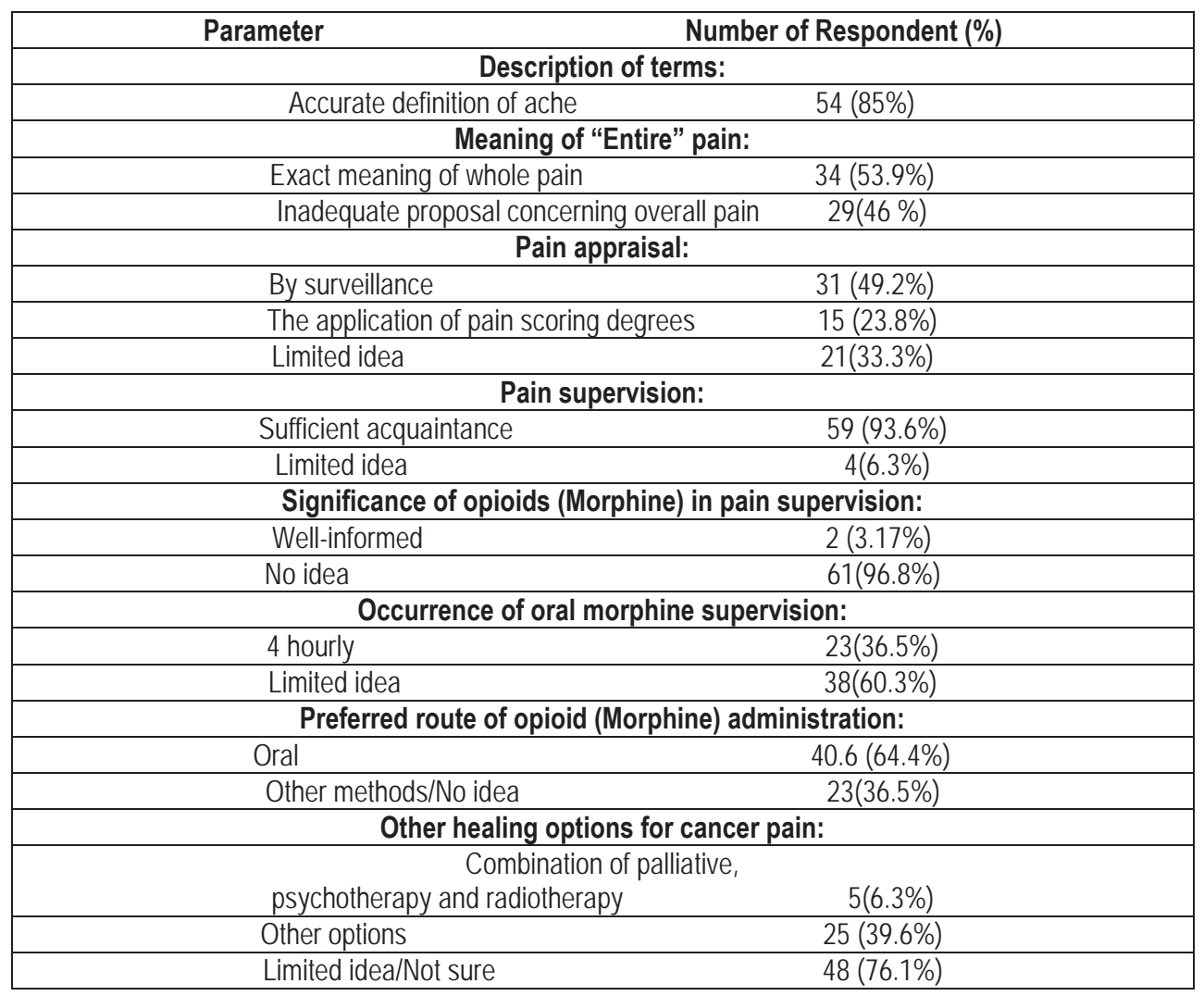


When requested to describe ache, 54 (85\%) participants got it correctly and $9(15 \%)$ had no idea. Beyond these hundred participants who described pain properly, just $3 \%$ really gave feature explanation on how ache could be administered. While required to classify entire pain, only 34 (53.9\%) could offer adequate meaning of "overall" ache, whereas 29(46\%) arise with diverse descriptions.

On ache appraisal method, $31(49.2 \%)$ of the participants said ache should be appraised by corporal observation of subjects; 15 (23.8\%) would apply pain ranking level; and 21(33.3\%) did not recognize what to accomplish. 59 (93.6\%) of the participants could categorize pain suitably.

While required regarding the application of opioids (morphine) in the administration of tumor pain, $61(96.8 \%)$ of the nurses did not even know the significance of opioids as powerful analgesics for harsh ache administration. 23(36.5\%) of the nurses recognized the gap for oral management morphine to be 4 hourly and 38(60.3\%) did not distinguish the proper interval. On the chosen itinerary of morphine management and its collateral results on the subject, just 40.6 (64.4\%) recognized the favorite method of managing and 56 (88.8\%) distinguished its collateral results on the patients. $21 \%$ of the respondents stated opioids should not be applied liberally for they are addictive, costly, very strong and out of stock, and they would not recommend morphine for subjects in ache.

When needed about tumor pain administration, 48 (76.1\%) had no idea; 25 (39.6\%) provided other alternatives and just $5(6.3 \%)$ affirmed a mixture of sedative, psychoanalysis and radiotherapy could be applied to handle tumor pain.

\section{Discussion}

Overall pain is described as the corporeal, common, emotional and spiritual ache a subject experiences. In this survey, just $24(38 \%)$ of the respondents could identify entire pain. It is the awareness of the nature of ache, obtained by the care supplier that would assure valuable administration of pain.

The best view to tumor ache management is during both pharmacologic and non-pharmacologic patterns. In this survey, just $22 \%$ of the participants were right.

Regarding World Health Organization (WHO), palliative management of ache which is a three-footstep issue, offers an helpful cure of ache at several grades of harshness (Bonica JJ 1985, Jadad AR, Browman GP 1995). This issue engages the application of non-opioid drugs like acetaminophen at the primary level. If the subject's ache still prolongs following recommendations, a weak opioid is begun besides the preceding drugs at the next stage.

For handling of declining pain, a feeble opioid drug is stopped and substituted with a powerful opioid at the third stage (Ventafridda V, Saita L, Ripamonti C. et al. 1985) .Some doctors appling the WHO principles are typically faced with the issue of choosing which drug, particularly the opioids, to begin with and at what amount when modifying to tough opioids. Once ache management becomes scarce, the doctor can apply an elevated tier for pain administration (Azevedo SL, Ferreira K, Kimula M, Jacobsen TM 2006).

Pursuing the progress in pharmacogenomics, it is gradually more obvious that genetics occupy an essential position in subject sensitivity to analgesic. If a subject does not react to a pain release mediator, the present medical schedule should be enhanced to highest tolerance amount or controlled to a diverse instrument. Nearly $68 \%$ to $82 \%$ of subjects comprising pain concerning tumor are handled with WHO pain administration approach (WHO guidelines cancer pain relief 1996; Palmer SN, Giesecke NM, Body SC. et al 2005).

A different dealing outline that could be managed for tumor pain management is the adjuvant treatment (Stamer UM, Bayerer B, Stuber F 2005, Ventafridda V et al 1985). This is started at any position of WHO palliative system as complement. Adjuvant treatment engages the aplication of drug adapted towards detailed causes of nociceptive ache, neuropathic ache, and diminution of opioid side consequences.

Neuropathic ache is usually not receptive to opioid drugs, except considerably elevated doses are utilized; medications demonstrated to be useful in the administration of neuropathic signs are Gabapentin, Carbamazepine (Zech DF, Grond S, Lynch J, et al 1995). These are categorized as anti-convulsants. Both Gabapentin and Pregabalin are expelled by renal path and have minimum drug - drug dealings. These make them valuable initial contour cure for neuropathic ache (Milch RA 2005).

Another category of medications that could be used to stabilize neurons involved in neuropathic pain is the antidepressants. Low doses of tricyclic anti-depressants such as Amitriptyline, has been shown to be effective for pain relief (Irving GA 2005). Other drugs that are rarely prescribed for neuropathic pain are serotonin re-uptake inhibitors and newer classes of anti-depressants such as selective serotonin inhibitors. Since most patients with neuropathic pain often have depressive or anxiety symptoms, anti-depressant medication with its potentials to treat both conditions, has shown

improved efficacy in the treatment of neuropathic pain. 
Insufficient awareness of analgesic medications managed to patients is one of the frequently mentioned motives for underneath cure of pain (Hainline B et al 2005). In this survey, $68 \%$ of the participants have heard about opioids, but not at all distinguished how it should be managed. This is reliable with preceding surveys where conclusions illustrated that there are considerable deficits on the universal submission of opioid medicine.

Opioid analgesics are the major medicines accustomed to control reasonable to harsh pain, mainly in tumor subjects. Despite their standard efficiency, opioids habitually are not generously accessible as a result of restraining regulations prior to the fright of mistreatment and violence.

Nursing workforce engage an essential place in the supervision of patients' ache and frequently, they are the primary to recognize the issue of unrestrained pain. In this survey, simply $17 \%$ of the nurses interrogated identified a ranking level should be applied to evaluate ache in patients previous to initiation of suitable administration.

In a survey by Bernardi $\mathrm{M}$ et al on a countrywide study of Italian hospital nurses, analysis of items demonstrated that more than $30 \%$ of hospital nurses undervalued the patients' ache and they did not took care of the ache in the accurate manner; they had a mistaken self-assessment regarding their ache administration awareness. Consequences from stepwise deterioration illustrated that nurses with elevated accurate response scores had focused more itineraries on pain instruction (Bernardi M \& Catania G 2007).

The attitude of the greater part of the nursing workforce is that palliative management should be focused on patient's demand and this confirmed that most skilled nurse-midwives do not distinguish the notion of stable state pain reliever, which guarantees that interminable dosing treatment, is directed to patient. The origin of this idea is that incapacity of patient to articulate ache or lodge a pain grumble does not signify absence of pain in that subject.

The fright of dependence stated by $21(28 \%)$ nurses in this survey as the cause why they would not generously manage opioids to administer tumor ache stays a substantial apprehension and a barrier to useful application of sedative analgesics. An analogous sensation was stated by $72 \%$ of the participants in Cohen's survey (Cohen F. L et al 1980). Likewise in a study by Schmidt et al examination of questionnaires from contributors who were pediatric nurses indicated that they were concerned for tumor patients frequently but had reduced comprehension of common standards of ache administration for the tumor subject.

Nurses had augmented apprehensions concerning the threat of dependence and respiratory dejection related to sedative analgesics (Schmidt K, Eland J 1994). In this survey, simply 38\% of the participants recognized suitable extent of management of oral fluid morphine whereas the mainstream did not distinguish. The knowledge was the same in the survey accomplished at the University Hospital Centre "Mother Theresa"Albania, where a major percentage of participants did not identify the proper extent of running opioids (Soyanwo $O$ 1999). The majority of the participants 42 $(66 \%)$ in this survey did not distinguish any additional technique for tumor pain organization separately to the application of analgesic. This signifies that they were not attentive of non-pharmacological resources of pain administration.

\section{Conclusion}

The results in this survey illustrated that the information of tumor pain appraisal and administration in nurses, stimulated with the task of caring for these subjects in different areas at the University Hospital Centre "Mother Theresa" is inadequate. This is perceptible to the information that the majority of them did not comprise official education in ache administration at the local or global level. A lot of of them are known with oral way of opioids management but the logistics of supervision is not obvious to them. In addition, a lot of them did not discern how to appraise tumor pain nor the patterns of healing to be applied.

\section{Recommendations}

With the aim of advancing the nursing care of tumor subjects in aches, recurrent official education in ache administration should be comprised in their medical practice. Formulate pain appraisal a part of essential marks to be examined. Involvement in workshop on diverse sorts of pain and its supervision should be a component of the endorsement implementation for nursing workforce. Besides these, pain administration should be included into the curriculum of Faculty of Technical Medical Sciences in order to generate potential nurses with significant comprehension of tumor ache management.

Furthermore, it is suggested that practitioners in Albania connect with government power to enhance understanding of the therapeutic requirement for opioids, highlighting that their absence of accessibility is the basis of harsh pain and affliction between tumor patients. 


\section{References}

Merskey and N. Bogduk 1994. IASP Press, Seattle; 209-14.

Besson JM, Chaouch A 2005. Peripheral and spinal mechanisms of nociception Physiol; 186.

Bonica JJ 1985. Treatment of cancer pain: current status and future needs. In: Fields, H.L. et.al. ed. Advances in pain research and therapy. Vol. 9. New York, Raven Press. 589-616.

Jadad AR, Browman GP 1995. The WHO analgesic ladder for cancer pain. WHO; 274: 1870-1873.

Ventafridda V, Saita L, Ripamonti C et al. 1985. WHO guidelines for the use of analgesics in cancer pain. Int. Jour. Tissue React;7: 9396.

Azevedo SL, Ferreira K, Kimula M, Jacobsen TM 2006. The WHO analgesic ladder for cancer pain control, twenty years of use. How much pain relief does one get from using it? Support Care Cancer.

World Health Organization. WHO guidelines cancer pain relief, 2nd ed. 1996. Geneva: World Health Organization.

Palmer SN, Giesecke NM, Body SC. et al 2005. Pharmacogenetics of anesthetic agents. Anesthesiology; 102: 663-671.

Stamer UM, Bayerer B, Stuber F 2005. Genetics and variability in opioid response. Eur J Pain; 9: 101-105.

Ventafridda V, Saita L, Ripamonti C. et al 1985. WHO guidelines for the use of analgesics in cancer pain. Int J Tissue React; 7: $93-96$.

Zech DF, Grond S, Lynch J, et al 1995. Validation of World Health Organization Guidelines for cancer pain relief: a 10-year prospective study. Pain; 63: 65-67.

Milch RA 2005 Neuropathic pain: implications for the surgeon. Surg Clin North Am; 85: 225-236.

Irving GA 2005. Contemporary assessment and management of neuropathic pain. Neurology; 64 (suppl 12 \& 13): 21-27.

Hainline B 2005. Chronic pain: physiological, diagnostic and management considerations. Psychiatr. Clin North Am; 28: $713-735$. Cancer Medicine 4th Edition, page 1363- 1864.

Rajagopal MR, Joranson DE, Gilson AM 2001. Medical use, misuse and diversion of opioids in India. Lancet; 358: 139-43.

Bernardi M, Catania G 2007. Knowledge and attitudes about cancer pain management: a national survey of Italian hospice nurses. $G$ cancer Nurs. Mar-Apr; 30(2): E20-6.

Cohen F. L 1980. Post surgical pain relief: Patients' status and nurses' medication pain; 9: 265-274.

Schmidt K, Eland J, Weiler K. J 1994. Pediatr Oncol Nurs. Pediatric cancer pain management: a survey of nurses' knowledge. Jan; 11(1): 4-12; discussion 13

Soyanwo O 1999. A post operative pain control prescripts pattern and patients experience. West African Journal Med ; 18: $207-210$. 